DAMTP/96-93, gr-qc/9610075

\title{
Comment on "Spacetime Information"
}

\author{
Adrian Kent* \\ Department of Applied Mathematics and Theoretical Physics, \\ University of Cambridge, \\ Silver Street, Cambridge CB3 9EW, U.K.
}

(18th February, 1997)

\begin{abstract}
A recent paper by Hartle [1] proposes a definition of "spacetime information" — the information available about a quantum system's boundary conditions in the various sets of decohering histories it may display — and investigates its properties. We note here that the analysis of Ref. [1] contains errors which invalidate several of the conclusions. In particular, the proof that the proposed definition agrees with the standard definition for ordinary quantum mechanics is invalid, the evaluations of the spacetime information for timeneutral generalized quantum theories and for generalized quantum theories with non-unitary evolution are incorrect, and the argument that spacetime information is conserved on spacelike surfaces in these last theories is erroneous. We show however that the proposed definition does, in fact, agree with the standard definition for ordinary quantum mechanics. Hartle's definition relies on choosing, case by case, a class of fine-grained consistent sets of histories. We supply a possible general definition of a class of sets that includes
\end{abstract}

*E-mail: apak@damtp.cambridge.ac.uk 
all the sets considered in Ref. [1] and that generalizes to other cases.

Typeset using REVTEX 
In a characteristically intriguing recent paper [四], Hartle investigates the problem of characterising the information available about the boundary conditions in generalized quantum theories in which the notion of a quantum state on a spacelike surface is not necessarily defined. Hartle's definition has three main ingredients: the standard definition for the missing information in a probability distribution, the formulation of quantum theory in terms of consistent sets of histories, and Jaynes' maximum entropy construction [2].

Specifically, Hartle considers quantum theories defined by general decoherence functionals $D$, which encode the boundary conditions and perhaps other information, and supposes that some natural standard class $\mathcal{C}_{\text {stand }}$ of consistent sets of histories has been fixed. He then defines the missing information in the boundary conditions to be

$$
\mathcal{S}(D)=\min _{\left\{C_{\alpha}\right\} \in \mathcal{C}_{\text {stand }}}\left[-\sum_{\alpha} p(\alpha) \log p(\alpha)\right],
$$

where $p(\alpha)$ is the probability of the history $C_{\alpha}$. Given this definition, Hartle proceeds to define the missing information in any consistent set of histories to be the maximum missing information in any set of boundary conditions which reproduce the decoherence functional matrix elements applied to the set:

$$
S\left(\left\{C_{\alpha}\right\}\right)=\max _{\tilde{D}}[\mathcal{S}(\tilde{D})]_{\tilde{D}\left(\alpha^{\prime}, \alpha\right)=D\left(\alpha^{\prime}, \alpha\right)} .
$$

The missing information in a class $\mathcal{C}$ of consistent sets of histories is then defined to be

$$
S(\mathcal{C})=\min _{\substack{\text { deconerent } \\\left\{C_{\alpha}\right\} \in \mathcal{C}}} S\left(\left\{C_{\alpha}\right\}\right) .
$$

Finally, the complete information available is defined to be the missing information in the class of all consistent sets of histories:

$$
S_{\text {compl }}=\min _{\text {decoherent }\left\{C_{\alpha}\right\}} S\left(\left\{C_{\alpha}\right\}\right) .
$$

Obviously, these definitions are open to criticism. Consistent sets of histories have some strange properties [3 [7] and it does not seem at all clear that they are the most natural objects to choose from within a spectrum which ranges, at least, from the class of all sets of 
quantum histories, through the sets of linearly positive histories $\mathbb{8}$, to the ordered consistent sets of histories [7]. It does not seem clear, either, that the maximum value of the missing information defined by any possible set of boundary conditions is a particularly useful measure of the missing information about the boundary conditions.

Hartle's proposals might thus perhaps best be seen as a pioneering attempt to investigate a question which is still in need of some elucidation. It would be interesting to understand more clearly what precisely it is that we are attempting to measure when we frame a definition of spacetime information, and (hence) what properties should or might be required of the measure. It may well be that there are several different natural measures, suited for different purposes. Indeed, Isham and Linden have recently proposed alternative definitions [9] which apply within their algebraic generalisation of the consistent histories formalism, while Gell-Mann and Hartle [10] and the author [11] have also investigated other possibilities.

Still, Hartle's proposed measure of missing information is a relatively simple quantity which gives at least some indication of how well the boundary conditions are constrained. It has also been investigated as a possible ingredient in a set selection mechanism [12,13]. In the remainder of this Comment we accept definitions (11 1 ) and examine their properties.

We need first to define the class $\mathcal{C}_{\text {stand }}$. After discussing two possible general definitions, neither of which seems to be completely satisfactory, in Section VI of Ref. [1], Hartle resorts to supplying definitions case by case. We would here like to suggest a new general definition that might be of use.

To define this class, we need to assume that the theory admits a natural class of completely fine-grained projective decompositions of the identity: that is, decompositions of the identity into orthogonal one-dimensional projections. Let us call these finest decompositions. The specific examples we have in mind here are sets of one-dimensional projections applied at any single time in a non-relativistic theory, such as standard quantum mechanics or the time-neutral quantum mechanics considered by Gell-Mann and Hartle [14], or applied on any spacelike hypersurface in a theory with fixed background geometry, such as those modelled by Anderson [15]. We need also to assume that at least one member of this class defines a 
consistent set of histories.

When these assumptions hold, as they do in the examples considered below, we propose to take $\mathcal{C}_{\text {stand }}$ to be the class of consistent sets of histories that are defined entirely by finest decompositions and that cannot consistently be extended by any further finest decompositions. This definition of $\mathcal{C}_{\text {stand }}$ includes all the consistent sets allowed by Hartle's definitions in the examples considered in Ref. [1]. It also applies, for example, to the case of a background geometry with more than one compact non-chronal region, for which no definition seems so far to have been suggested. However, as we will see below, it also includes consistent sets disallowed by Hartle's definitions in the case of a background geometry with a non-chronal region.

We turn now to Hartle's analysis of the properties of definitions (11). It is helpful to begin with the discussion of time-neutral generalized quantum mechanics in Section V, since this causes difficulties elsewhere in the paper. Following the above scheme, Hartle defines

$$
\mathcal{S}\left(\tilde{\rho}^{f}, \tilde{\rho}^{i}\right) \equiv \underset{\substack{\text { fine-grained } \\ \text { decoherent }\left\{C_{\alpha}\right\}}}{\min }\left[-\sum_{\alpha} p(\alpha) \log p(\alpha)\right]
$$

where the minimum is over the completely fine-grained decoherent sets $\left\{C_{\alpha}\right\}$ for which

$$
D\left(\alpha^{\prime}, \alpha\right)=\mathcal{N} \operatorname{Tr}\left(\tilde{\rho}^{f} C_{\alpha^{\prime}} \tilde{\rho}^{i} C_{\alpha}^{\dagger}\right)=\delta_{\alpha^{\prime} \alpha} p(\alpha)
$$

The completely fine-grained histories consist of sequences of sets of one-dimensional projections at each and every time, but since repeating the same set of projections has no effect on the entropy, only the distinct projective decompositions in any given consistent set need be considered.

Hartle argues that, since non-trivial consistent extensions increase the value of $-\sum_{\alpha} p(\alpha) \log p(\alpha)$, the minimum is attained by a set of histories of the form

$$
C_{\alpha}=P_{\alpha_{f}}^{f} P_{\alpha_{i}}^{i}
$$

where $P_{\alpha_{f}}^{f}$ are projections onto a basis $\left\{\left|\alpha_{f}\right\rangle\right\}$ diagonalizing $\tilde{\rho}^{f}$ and $P_{\alpha_{i}}^{i}$ are projections onto a basis $\left\{\left|\alpha_{i}\right\rangle\right\}$ diagonalizing $\tilde{\rho}^{i}$. 
This is wrong, for two reasons. First, the increasing entropy argument, given by Hartle in equations (5.8)-(5.10), actually implies that the minimum is attained by histories containing a single projective decomposition, repeated at all times, rather than two different decompositions. Second, although the diagonalization of $\tilde{\rho}^{i}$ and $\tilde{\rho}^{f}$ by the relevant projections is a sufficient condition for consistency, it is not necessary. The minimum is attained by a set of histories of the form

$$
C_{\alpha}=P_{\alpha}
$$

and in general the projections $P_{\alpha}$ need not diagonalize either $\tilde{\rho}^{i}$ or $\tilde{\rho}^{f}$. The consistency condition

$$
\left\langle\alpha\left|\tilde{\rho}^{i}\right| \alpha^{\prime}\right\rangle\left\langle\alpha^{\prime}\left|\tilde{\rho}^{f}\right| \alpha\right\rangle=\operatorname{Tr}\left(\tilde{\rho}^{f} P_{\alpha} \tilde{\rho}^{i} P_{\alpha^{\prime}}\right)=0 \quad \text { if } \alpha \neq \alpha^{\prime}
$$

requires only that for each pair of distinct $\alpha, \alpha^{\prime}$ either $\left\langle\alpha\left|\tilde{\rho}^{i}\right| \alpha^{\prime}\right\rangle$ or $\left\langle\alpha\left|\tilde{\rho}^{f}\right| \alpha^{\prime}\right\rangle$ should vanish. There are generally many bases with this property. For example, we could choose a basis $\left\{\left|\alpha_{i}\right\rangle\right\}$ by taking $\left|\alpha_{1}\right\rangle$ to be an eigenvector of $\tilde{\rho}^{i},\left|\alpha_{2}\right\rangle$ to be an eigenvector of the matrix obtained by restricting $\tilde{\rho}^{f}$ to the subspace orthogonal to $\left|\alpha_{1}\right\rangle,\left|\alpha_{3}\right\rangle$ to be an eigenvector of the matrix obtained by restricting $\tilde{\rho}^{i}$ to the subspace orthogonal to $\left|\alpha_{1}\right\rangle$ and $\left|\alpha_{2}\right\rangle$, and so on.

Most of the discussion in the remainder of Section V of Ref. [1] is therefore incorrect.

There seems to be an additional problem later in this section. Hartle observes that

$$
S_{\mathrm{compl}} \geq \mathcal{S}\left(\rho^{f}, \rho^{i}\right)
$$

by definition, and then goes on to suggest that the equation is an equality when $\rho^{i}$ and $\rho^{f}$ are non-degenerate. The condition required for this argument to go through is that the decoherence matrix elements of a consistent set of histories of the form (7) should determine the operators $\rho^{i}$ and $\rho^{f}$ up to rescalings, not that the operators should determine the set, so that the degeneracy of $\rho^{i}$ and $\rho^{f}$ seems to be irrelevant. It is, however, true that (10) is generically an equality. A sufficient condition for $\rho^{i}$ and $\rho^{f}$ to be determined up to rescalings, which generically holds true, is that there exist bases $\left\{\left|\alpha_{i}\right\rangle\right\}$ and $\left\{\left|\beta_{i}\right\rangle\right\}$ diagonalizing $\rho^{i}$ and 
$\rho^{f}$ respectively, with the property that $\left\langle\alpha_{i} \mid \beta_{j}\right\rangle \neq 0$ for all $i, j$. When these bases exist, the corresponding fine-grained projective decompositions define the desired consistent set.

It follows that, generically, it is true that complete information is available about the initial and final conditions on any pair of separated spacelike surfaces, as Hartle suggests in section V.D. It does not seem immediately obvious, though, that complete information is generically not available on a single spacelike surface, as Hartle suggests, although it seems usually to be true that a single spacelike surface will not suffice.

The discussion of spacetime information in standard quantum mechanics, which follows and relies on equation (5.13), is also invalid. To understand the point of this discussion, note that, while the aim of Ref. [1] is to define a measure of spacetime information from first principles, the standard entropy functional,

$$
\mathcal{S}(\tilde{\rho})=-\operatorname{Tr}(\tilde{\rho} \log \tilde{\rho})
$$

for a single density matrix is taken for granted in the text up to equation (5.16). Hartle notes this when introducing the entropy functional in equation (2.6), and promises there a justification from a more general point of view in Section V. It is this justification which equations (5.13)-(5.16), and the accompanying text, are intended (inter alia) to supply. Hartle concludes, after equation (5.16), that the standard entropy functional on single density matrices has been derived as the least missing information in fine-grained decoherent sets of histories.

In this discussion, Hartle considers the case when $\tilde{\rho}^{f}=I$ and $\tilde{\rho}^{i} \equiv \tilde{\rho}$, and assumes that the fine-grained projective decompositions which define consistent sets are precisely those which diagonalize the initial density matrix $\tilde{\rho}$.

In fact, any projective decomposition at a single time defines a consistent set for these boundary conditions, and in particular all fine-grained projective decompositions define consistent sets. The minimization therefore needs to be carried out over all fine-grained projective decompositions.

In other words, the proof of another of the key claims of Ref. [1], namely the derivation 
of the usual information measure for single density matrices from Hartle's general definition,

$$
\mathcal{S}_{\text {usual }}(\tilde{\rho})=-\operatorname{Tr}(\tilde{\rho} \log \tilde{\rho})=\mathcal{S}_{\text {Hartle }}(\tilde{\rho}),
$$

fails. It is, however, true that the two measures agree, as we now show. (We generally write $\mathcal{S}(\tilde{\rho})$ for both these measures, since they are equal, but have added labels here and below for clarity.)

According to the general definition of Ref. [1], the missing information for a standard quantum theory with the initial state $\tilde{\rho}$ is

$$
\mathcal{S}_{\text {Hartle }}(\tilde{\rho}) \equiv \min _{\substack{\text { fine-grained } \\ \text { decoherent }\left\{C_{\alpha}\right\}}}\left[-\sum_{\alpha} p(\alpha) \log p(\alpha)\right]
$$

where the minimum is over the completely fine-grained consistent sets $\left\{C_{\alpha}\right\}$ for which

$$
D\left(\alpha^{\prime}, \alpha\right)=\mathcal{N} \operatorname{Tr}\left(C_{\alpha^{\prime}} \tilde{\rho} C_{\alpha}^{\dagger}\right)=\delta_{\alpha^{\prime} \alpha} p(\alpha)
$$

The increasing entropy argument means that it is sufficient to take the minimum over consistent sets defined by fine-grained projective decompositions $\left\{P_{\alpha}\right\}$. We therefore need to show that

$$
\min _{\text {fine-grained }\left\{P_{\alpha}\right\}}\left[-\sum_{\alpha} p(\alpha) \log p(\alpha)\right]=-\operatorname{Tr}(\tilde{\rho} \log \tilde{\rho}) \text {. }
$$

Consider any fine-grained projective decomposition $\left\{P_{\alpha}\right\}$, write $\tilde{\rho}=(\tilde{\rho})_{\alpha \beta}$ as a matrix in the basis defined by $\left\{P_{\alpha}\right\}$, and define the density matrix $\tilde{\rho}^{\prime}$ in the same basis by

$$
\left(\tilde{\rho}^{\prime}\right)_{\alpha \beta}= \begin{cases}(\tilde{\rho})_{\alpha \beta} & \text { for } \alpha=\beta \\ 0 & \text { for } \alpha \neq \beta\end{cases}
$$

We have that $(\tilde{\rho})_{\alpha \alpha}=\left(\tilde{\rho}^{\prime}\right)_{\alpha \alpha}=p(\alpha)$, so that

$$
-\operatorname{Tr}\left(\tilde{\rho} \log \tilde{\rho}^{\prime}\right)=-\sum_{\alpha} p(\alpha) \log p(\alpha)
$$

Now

$$
-\operatorname{Tr}\left(\tilde{\rho} \log \tilde{\rho}^{\prime}\right) \geq-\operatorname{Tr}(\tilde{\rho} \log \tilde{\rho})
$$


holds [1,16] for all density matrices $\tilde{\rho}$ and $\tilde{\rho}^{\prime}$. On the other hand, when the decomposition $\left\{P_{\alpha}\right\}$ diagonalizes $\tilde{\rho}$, we have that $\tilde{\rho}=\tilde{\rho}^{\prime}$ and hence that

$$
-\operatorname{Tr}\left(\tilde{\rho} \log \tilde{\rho}^{\prime}\right)=-\operatorname{Tr}(\tilde{\rho} \log \tilde{\rho})
$$

Thus (12) holds, as claimed.

For completeness we note also that, in the discussion of standard quantum mechanics in Section II of Ref. [1], equations (2.21) and (2.22) are unnecessary to establish (2.23), which holds by definition.

We turn now to the discussion of Section VII of Ref. [1], which considers Anderson's proposal [15] for modelling generalized quantum theories in background spacetimes with a non-chronal region by a decoherence functional which incorporates non-unitary evolution:

$$
D\left(\beta^{\prime}, \alpha^{\prime} ; \beta, \alpha\right)=\operatorname{Tr}\left[X^{-1} C_{\beta^{\prime}} X C_{\alpha^{\prime}} \rho C_{\alpha}^{\dagger} X^{\dagger} C_{\beta}\left(X^{\dagger}\right)^{-1}\right]
$$

where the non-unitary operator $X$ is assumed invertible.

We first comment briefly on the definition of the class $\mathcal{C}_{\text {stand }}$. Hartle proposes that in this case $\mathcal{C}_{\text {stand }}$ should consist of all consistent sets that are defined by fine-grained projective decompositions and that include at least one decomposition both before and after the nonchronal region. The increasing entropy argument means that, given this definition, it suffices to consider sets of histories of the form

$$
C_{\alpha}=P_{\alpha}^{i}=|\alpha\rangle\left\langle\alpha\left|; \quad C_{\beta}=P_{\beta}^{f}=\right| \beta\right\rangle\langle\beta|,
$$

where $\{|\alpha\rangle\}$ and $\{|\beta\rangle\}$ are bases.

The general definition of $\mathcal{C}_{\text {stand }}$ proposed above includes all the histories of the form (21), where the projections are repeated on a maximal collection of spacelike surfaces before and after the non-chronal region. However, it also allows fine-grained sets of histories all of whose projections are before the non-chronal region, provided that these sets have no extension by fine-grained projective decompositions after the region, and vice versa. It might perhaps be thought desirable to exclude such sets. On the other hand, the definition has the virtue 
of extending to the case of a spacetime with several non-chronal regions, whereas it may not generally be possible to find any consistent set which includes fine-grained projective decompositions in all the separate chronal regions.

We now consider Hartle's analysis, using the definition of $\mathcal{C}_{\text {stand }}$ proposed in Ref. [1]. To the extent that Hartle's discussion relies on the arguments of Section V, it is invalid. For instance, it is not always true that the histories of the form (21), in which the bases diagonalize $\rho$ and $X X^{\dagger}$ respectively, are adequate for the purpose of computing $S_{X}(\rho)$. To give a simple example, if $\rho=\left|\alpha_{1}\right\rangle\left\langle\alpha_{1}\right|$ is pure, and we take $\left|\beta_{1}\right\rangle$ to be $a X\left|\alpha_{1}\right\rangle$, where $a$ is a normalising factor, then any orthonormal bases $\left\{\left|\alpha_{i}\right\rangle\right\}$ and $\left\{\left|\beta_{i}\right\rangle\right\}$ that include $\left|\alpha_{1}\right\rangle$ and $\left|\beta_{1}\right\rangle$ define a consistent set of the form (21). Any set defined in this way has history probabilities with zero entropy, since all the histories except one have zero probability. On the other hand, for general $X$, all sets defined by bases diagonalizing $\rho$ and $X X^{\dagger}$ have history probabilities with positive entropy.

The discussion of spacetime information on single surfaces also contains errors. Equation (7.15) in Ref. [1] is incorrect, and should be replaced by

$$
\begin{aligned}
\tilde{D}\left(\alpha^{\prime}, \alpha\right) & =\operatorname{Tr}\left[X^{-1} X P_{\alpha^{\prime}} \tilde{\rho} P_{\alpha} X^{\dagger}\left(X^{-1}\right)^{\dagger}\right] \\
& =\left\langle\alpha^{\prime}|\tilde{\rho}| \alpha\right\rangle\left\langle\alpha \mid \alpha^{\prime}\right\rangle=D\left(\alpha^{\prime}, \alpha\right)=\left\langle\alpha^{\prime}|\rho| \alpha\right\rangle\left\langle\alpha \mid \alpha^{\prime}\right\rangle
\end{aligned}
$$

Clearly, it is not true that, as Hartle suggests, $\tilde{\rho}=\rho$ is the unique density matrix which reproduces the decoherence functional. This means that Hartle's evaluation of the information available on spacelike surfaces before the non-chronal region is invalid, so that his argument for the equality (7.13) fails. It follows from Hartle's equations (7.17) and (7.18), however, that the equality (7.13) is, at least, generically true, assuming (as Hartle does) that the operator $X$ is known a priori.

The argument for another key claim of Ref. [1], that the information is conserved on spacelike surfaces on either side of the non-chronal region, likewise fails, and it does not seem obvious that this claim is generally true. 


\section{ACKNOWLEDGMENTS}

I would like to thank Chris Isham, Noah Linden and Jim McElwaine for helpful discussions and Jim Hartle for a critical reading of the manuscript and useful comments. This work was supported by a Royal Society University Research Fellowship. 


\section{REFERENCES}

[1] J.B. Hartle, Phys. Rev. D 51, 1800 (1995).

[2] See, for example, R.D. Rosenkrantz, ed. E.T. Jaynes: Papers on Probability Statistics and Statistical Mechanics, (D. Reidel, Dordrecht, 1983).

[3] F. Dowker and A. Kent, Phys. Rev. Lett. 75, 3038 (1995).

[4] F. Dowker and A. Kent, J. Stat. Phys. 82, 1575 (1996).

[5] A. Kent, Phys. Rev. A 54, 4670 (1996).

[6] A. Kent, gr-qc/9604012, to appear in Phys. Rev. Lett.

[7] A. Kent, gr-qc/9607073, submitted to Ann. Phys., University of Cambridge preprint DAMTP/96-74.

[8] S. Goldstein and D.N. Page, Phys. Rev. Lett. 74, 3715 (1995).

[9] C. Isham and N. Linden, quant-ph/9612035, to appear in Phys. Rev. A.

[10] J. Hartle, private communication.

[11] A. Kent, unpublished.

[12] M. Gell-Mann and J.B. Hartle, in Complexity, Entropy and the Physics of Information, Vol. III of SFI Studies in the Science of Complexity, ed. by W. Zurek (Addison Wesley, Reading, 1990).

[13] J.N. McElwaine, Approximate Consistency and Prediction Algorithms in Quantum Mechanics, University of Cambridge Ph.D. thesis (1996).

[14] M. Gell-Mann and J.B. Hartle in Proceedings of the NATO Workshop on the Physical Origins of Time Asymmetry, Mazagón, Spain, September 30-October 4, 1991, ed. by J. Halliwell, J. Pérez-Mercader, and W. Zurek, (Cambridge University Press, Cambridge, 1994). 
[15] A. Anderson, Phys. Rev. D 51, 5707 (1995).

[16] See, for example, O. Bratteli and D.W. Robinson, Operator Algebras and Quantum Statistical Mechanics, (Springer Verlag, New York, 1981). 